\title{
Belgeo
}

Revue belge de géographie

4 | 2004

Maritime and port economic geography

\section{Quelques conséquences de l'arrivée prochaine des méga porte-conteneurs}

Some consequences of the growing use of mega containerships

Jacques Marcadon

\section{CpenEdition}

Journals

Édition électronique

URL : http://journals.openedition.org/belgeo/13489

DOI : 10.4000/belgeo.13489

ISSN : 2294-9135

Éditeur :

National Committee of Geography of Belgium, Société Royale Belge de Géographie

Édition imprimée

Date de publication : 31 décembre 2004

Pagination : 407-432

ISSN : 1377-2368

Référence électronique

Jacques Marcadon, «Quelques conséquences de l'arrivée prochaine des méga porte-conteneurs »,

Belgeo [En ligne], 4 | 2004, mis en ligne le 15 septembre 2013, consulté le 01 mai 2019. URL : http:// journals.openedition.org/belgeo/13489; DOI : 10.4000/belgeo.13489

Ce document a été généré automatiquement le 1 mai 2019.

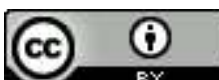

Belgeo est mis à disposition selon les termes de la licence Creative Commons Attribution 4.0 International. 


\section{Quelques conséquences de l'arrivée prochaine des méga porte- conteneurs}

Some consequences of the growing use of mega containerships

Jacques Marcadon

1 Le développement des porte-conteneurs post-panamax a débuté au milieu des années 1980, mais ce n'est que plus de dix ans plus tard que l'on commence à parler de l'entrée en flotte des 8000 EVP (Equivalent Vingt Pieds). Là une limite est atteinte par la taille et la puissance des moteurs diesel. L'armement Maersk va modifier en 1997 ses porteconteneurs de la classe $\mathrm{K}$, ceux de $6000 \mathrm{EVP}$ en leur ajoutant 2 rangées de boîtes, créant ainsi la classe S transportant plus de $7000 \mathrm{EVP}$. C'est en 2002 que les premiers $8000 \mathrm{EVP}$ entrent en service. Des plans existent pour construire des navires de 15000 à 18000 EVP.

2 L'article se propose de montrer dans une première partie la tendance forte à l'utilisation de TGPC (Très Grands Porte-Conteneurs) en liaison avec les besoins accrus d'échanges.

3 La seconde partie examine les conséquences sur les marchandises transportées et l'obligation de remplir les boîtes. Enfin, du fait des limites techniques des terminaux actuels pour l'accueil des TGPC, il est fait état des nouveaux concepts et des projets dans le domaine portuaire. Du fait du cadre limité de cet article, le rôle des chargeurs est volontairement occulté, l'accent est mis principalement sur le navire et les armateurs.

De nombreux auteurs se sont interrogés sur les conséquences de la conteneurisation dans différents domaines, armatorial, portuaire (Genet, 1999 ; Maas, 2001 ; Musso et al., 1999 ; Notteboom, 2002...), le sujet n'est pas nouveau, mais ici avec l'arrivée des TGPC, un seuil quantitatif est franchi, un fait de rupture est atteint, d'où l'intérêt d'en examiner certaines conséquences, celles qui concernent plus directement les armements et celles qui concernent les ports. 


\section{La tendance à la construction de porte-conteneurs de plus en plus grands}

5 La tendance à aligner des porte-conteneurs de plus en plus grands s'appuie sur la croissance continue des volumes de marchandises conteneurisées (Darche, 2002) sur pratiquement toutes les grandes routes maritimes, une croissance plus forte pouvant être enregistrée dans certaines régions, comme c'est le cas en sortie d'Asie. Seulement la construction de navires de grande taille comme celle des porte-conteneurs de $15000 \mathrm{EVP}$ suppose résolus certains problèmes techniques.

\section{Les limites techniques au niveau de la construction ne sont pas un obstacle}

Entre autres, la puissance nécessaire de l'appareil propulsif a longtemps représenté une contrainte.

7 Les plus grands moteurs diesel ont une puissance maximum de $68000 \mathrm{Kw}$ fournissant assez de puissance à un porte-conteneurs post-panamax de $8000 \mathrm{EVP}$ afin d'atteindre une vitesse de 25 noeuds. Pour de plus grands navires, 2 moteurs seront nécessaires. Il existe une limite théorique au coût que représente l'usage de 2 moteurs en matière d'économie d'échelle car la consommation en carburant augmente d'environ $30 \%$ pour une vitesse accrue de $10 \%$.

8 Techniquement, les chantiers navals aptes à construire des VLCC (Very Large Crude Carriers) et des ULCC (Ultra Large Crude Carriers) ont les installations d'une capacité suffisante pour construire ces TGPC. Ils pourront engainer le canal de Suez avec une largeur utile de $64 \mathrm{~m}$ (210 feet). Mais les ingénieurs navals auront à réfléchir au design et à la stabilité du navire, tenus à ne pas dépasser une trop grande largeur du fait des possibilités des portiques de manutention, ce qui pose le problème de terminaux adaptés comme nous le verrons ultérieurement.

\section{Les commandes actuelles portent aussi bien sur les TGPC que sur les navires de taille moyenne}

Les commandes actuelles portent sur des navires de plus de 5000 EVP mais aussi sur des porte-conteneurs plus "petits» de 2500 à 4000 EVP car les armateurs ont besoin de navires de taille moyenne ou petite pour le feedering, les liaisons régionales et aussi certaines liaisons intercontinentales en trafic Nord-Sud en particulier.

10 S'il y a une forte croissance de la capacité de transport avec des navires de plus de 5000 EVP, ceux de 1000 à 4000 EVP représentent $60 \%$ des capacités fin 2002 (tableaux 1 et 2). Le constat est cependant sans ambiguïté : alors que le nombre de porte-conteneurs de 5000 EVP et plus était de 137 à la fin de 2001, il va atteindre 268 fin 2003. 
Tableau 1. Répartition de la flotte mondiale de porte-conteneurs en 2002.

\begin{tabular}{lcc}
\hline Classes de capacité & \% du nombre de navires & \% de la capacité en EVP \\
\hline $0-999$ EVP & 44 & 12 \\
$1000-3$ 999 EVP & 47 & 61 \\
$4000-5$ 999 EVP & 8 & 23 \\
Plus de 6 000 EVP & 1 & 4 \\
\hline Total & 100 & 100 \\
\hline
\end{tabular}

Source : Isemar d'après données Inrets - 2002

Tableau 2. Les commandes de porte-conteneurs en novembre 2002.

\begin{tabular}{llll}
\hline Armateurs (rang) & $1000-4000$ EVP & $4000-6000$ EVP & $>6000$ EVP \\
\hline Maersk-Sealand (1) & $1 \times 862 ; 6 \times 2500$ & $4 \times 4300$ & $1 \times 6600 ; 6 \times 10000$ \\
P\&O Nedlloyd (2) & $2 \times 1700 ; 7 \times 2500$ & $2 \times 4100$ & \\
Evergreen (3) & $2 \times 1600$ & & \\
Hanjin (4) & & $1 \times 4200$ & \\
MSC (5) & & $6 \times 4900 ; 4 \times 5050 ;$ & $1 \times 6400 ; 3 \times 6300$ \\
CMA - CGM (8) & $4 \times 2200$ & $8 \times 5800$ & $6 \times 6200 ; 3 \times 6300$ \\
NYK (9) & $1 \times 1200$ & & $6 \times 7700$ \\
CP Ships (10) & $3 \times 3200$ & $1 \times 4100$ & \\
OOCL (12) & $4 \times 2700$ & $8 \times 4500$ & \\
MOL (13) & & $5 \times 4000 ; 9 \times 4050 ;$ & \\
China Shipping (15) & & $5 \times 4800 ; 8 \times 5600$ & \\
Hapag Lloyd (16) & & & \\
Zim (18) & $4 \times 3100$ & 66 navires & 32 navires \\
CSAV (19) & 34 navires & & \\
\hline Total & & & \\
\hline
\end{tabular}

Source : Fairplay Weekly

11 Avec la croissance des échanges inter-régionaux en Europe, en Asie, en Amérique latine $\mathrm{du}$ fait des processus comme celui du Mercosur par exemple, c'est la taille moyenne de navires qui convient le mieux. Ainsi, si Maersk-Sealand est acteur dans les commandes de très grands porte-conteneurs, il a une flotte de 84 navires de 1000 à 2000 EVP. L'utilisation des très grands porte-conteneurs est nécessairement liée à l'utilisation de navires plus petits, comme dans le domaine militaire un porte-avions a besoin d'une flottille d'accompagnement pour être efficace. Les très grands porte-conteneurs ne peuvent se passer des plus petits navires, alors que ces mêmes petits navires peuvent être autonomes sur le marché.

12 Rappelons que lorsque furent lancées les premières lignes tour-du-monde deux stratégies étaient en oeuvre, celle d'Evergreen avec une double rotation vers l'Est et vers l'Ouest avec des porte-conteneurs de $200 \mathrm{EVP}$, alors que les USL (United States Lines) lançaient dans un seul sens des plus de 4000 EVP qui n'ont jamais atteint un taux de remplissage minimum; ce service a dû s'arrêter au bout de quelques mois. L'arrivée prochaine des TGPC n'est donc pas sans conséquences pour les armements maritimes. 


\section{Les conséquences pour les armements : le risque de surcapacité et les recompositions dans les systèmes d'alliances}

13 La surcapacité induite par l'arrivée de nouveaux volumes de cales va provoquer une baisse des taux de fret. L'économie d'échelle réalisée est-elle significative?

14 L'étude d'Isemar (Lopez Ponton, 2003) cite les travaux de plusieurs auteurs qui montrent la difficulté de réaliser des économies d'échelle en augmentant la taille des navires. Le passage de 1000 à 2000 EVP permet une économie d'échelle de $20 \%$ du coût du transport. Passer de 2000 à 4000 EVP réduit les coûts de $7 \%$ seulement, et passer de 4000 à 6000 EVP de $4 \%$. C'est le taux de remplissage, la croissance du volume transporté qui comptent le plus, davantage que la taille même du navire. A partir d'un certain seuil, les très grands navires ne génèrent pas autant d'économies, surtout si le contexte général des échanges internationaux n'est pas porteur.

Après la crise asiatique de 1997-1998 qui avait affecté les échanges conteneurisés, la reprise de 1999-2000 a incité les armements à persévérer dans la commande de porteconteneurs de plus en plus grands, alors que peu nombreux étaient les navires envoyés à la démolition.

16 La capacité de la flotte de porte-conteneurs de plus de 1000 EVP a atteint fin $20015,3 \mathrm{M}$ EVP (+ 12,7\% sur l'année précédente), $6 \mathrm{M}$ EVP fin $2002(+14 \%)$ et les estimations pour 2003 (+ $9 \%$ à nouveau) donnent un chiffre de 6,5 M EVP (comparé à 2,6 M EVP en 1995). Or les volumes de fret n'augmentent qu'à hauteur de $6 \%$ à $7 \%$ l'an environ.

17 Parallèlement avec une économie léthargique depuis les attentats du 11 septembre 2001 et l'éclatement de la bulle financière de la nouvelle économie, la surcapacité est réelle et les taux de fret très bas ne couvrent qu'à peine les coûts du transport. La politique des armements est de désarmer des navires. Mais le désarmement d'un porte-conteneurs de 6000 à 7000 boîtes coûte cher, et ce sera pire pour un TGPC. Les exemples récents sont là pour montrer la nécessité de désarmer des navires dans l'attente de la remontée des taux de fret: en janvier 2002 la «Grand Alliance » avait immobilisé ainsi 10 navires. Au printemps 2002, Cosco, K-Line et Yang Ming, qui ont constitué l'Atlantic Space Charter Agreement, ont retiré une rotation hebdomadaire sur les 5 prévues avec des navires de 2000 EVP.

18 Est-ce que les armements vont continuer à retirer volontairement leurs navires dans les années à venir dans la perspective de l'offre de cale supplémentaire représentée par l'arrivée des TGPC ? La discipline entre les lignes et la coopération ne sont pas faciles à organiser d'autant que les organes régulateurs aux Etats-Unis et en Europe surveillent les rapprochements entre transporteurs. Les risques de déstabilisation du marché sont réels et à tout le moins des recompositions dans les associations d'armements et alliances sont à prévoir. L'investissement considérable qu'implique la mise en flotte des navires géants risque de modifier le paysage des regroupements de compagnies, certaines ne survivront pas.

Davantage de fusions sont constatées actuellement, plus que des achats de compagnies car les armements ont du mal à trouver suffisamment de fonds pour financer des acquisitions (Alix, Slack, Comtois, 1999). La construction des navires géants qui vont coûter encore plus cher que les générations antérieures de porte-conteneurs va, d'une part, conduire à une sélection des armements ou groupes d'armements capables d'assurer 
le financement, et va d'autre part susciter des partenariats nouveaux au niveau des Alliances selon les intérêts en jeu. Des rapprochements entre grands armements sont prévisibles hors des Alliances également car l'Alliance est une structure contraignante, chaque partenaire est tenu par le poids des compromis. Les aléas de la demande et les variations des taux de fret ajoutés à la nécessité de remplir des cellules sur des navires de plus en plus grands poussent à des accords de coopération entre les armements bien que les partenaires ne respectent pas toujours ces accords en fonction de leurs intérêts.

L'arrivée des TGPC va créer des déséquilibres qui conduisent à la domination des plus forts plus qu'à la coopération.

21 La cellule coûtera moins cher sur les TGPC, mais l'effet sera très faible sur le client car le coût du transport maritime seul ne représente guère plus que $20 \%$ du prix total de la chaîne du transport. Ainsi le prix du transport d'une bouteille de whisky entre le Royaume-Uni et le Japon ne représente que 4 cents par bouteille. La crise actuelle de l'économie mondiale rend peu attractifs ces énormes porte-conteneurs, d'autant que les problèmes logistiques induits les rendent moins flexibles que des navires de taille inférieure. Mais à moyen et long terme, leur arrivée est inéluctable car il n'est pas dans la nature humaine de freiner un mouvement expansionniste.

L'intérêt pour les armateurs d'aligner de grands porte-conteneurs est d'obtenir des économies d'échelle par la réduction des frais fixes par cellule (frais de ports, coût d'exploitation et de capital), mais les coûts liés à la marchandise, a contrario, peuvent augmenter (manutention, assurances), alors que le problème du repositionnement des conteneurs s'accroît. De même, les frais administratifs qui sont proportionnels aux volumes transportés augmentent. Le temps de déchargement dans les ports et la congestion vont s'accroître, les systèmes de gestion des conteneurs et de leur acheminement terrestre devront être encore plus performants. Les autres partenaires de la filière de transport devront évoluer à leur tour pour suivre la massification induite par les armements maritimes (Slack, Comtois, Sletmo, 1996). Deux aspects contraignants, notamment, s'imposent aux armateurs des TGPC, la nécessité de remplir les boîtes et la gestion optimale de leur positionnement.

\section{Gagner des parts de marché de vracs}

L'arrivée des méga-porte-conteneurs est prévisible à terme, en dépit des contraintes énumérées précédemment et des vicissitudes économiques car la conteneurisation touche de plus en plus de marchandises transportées jusque-là en conventionnel, déchets, produits chimiques, malt, pierres, néo-vracs... La croissance des trafics portuaires conteneurisés est une réalité, certains ports notamment en Asie (Body, 2000 ; Cai, 2002 ; Numan, 2001; Neumeister, 2001) affichent des taux remarquables de plus de $33 \%$ d'une année à l'autre (tableau 3). 
Tableau 3. Classement des ports mondiaux selon le taux de croissance 1999-2000 du trafic de conteneurs.

\begin{tabular}{llcc}
\hline Rang & Port/Pays & Taux (\%) & Trafic 2000 (M EVP) \\
\hline 1 & Tanjung Priok/ Indonésie & 59,0 & 3,369 \\
2 & Qingdao / Chine & 36,4 & 2,100 \\
3 & Shenzen / Chine & 33,8 & 3,993 \\
4 & Shanghai / Chine & 33,1 & 5,612 \\
5 & Sydney / Australia & 33,0 & 1,016 \\
6 & Los Angeles / USA & 27,4 & 4,879 \\
7 & Port Klang / Malaisie & 25,7 & 3,206 \\
8 & Bremen-Bremerhaven / Allemagne & 24,4 & 2,712 \\
9 & Nagoya / Japon & 22,3 & 1,904 \\
10 & Laem Chabang / Thailande & 20,0 & 1,828 \\
11 & HongKong / Chine & 20,0 & 18,098 \\
12 & Gioia Tauro / Italie & 17,7 & 2,653 \\
13 & Keelung / Taïwan & 17,3 & 1,955 \\
14 & Pusan / Corée du Sud & 17,1 & 7,540 \\
15 & Algésiras / Espagne & 15,0 & 2,009 \\
16 & San Juan / Porto Rico & 14,8 & 2,393 \\
17 & Melbourne / Australie & 14,6 & 1,290 \\
18 & Hambourg / Allemagne & 13,6 & 4,248 \\
19 & Anvers / Belgique & 13,4 & 4,100 \\
20 & Barcelone / Espagne & 12,4 & 1,388 \\
21 & Valence / Espagne & 11,8 & 1,308 \\
22 & Tokyo / Japon & 9,8 & 2,960 \\
23 & Vancouver / Canada & 9,0 & 1,163 \\
24 & Dubail / Emirats Arabes Unis & 7,5 & 3,059 \\
25 & Singapour / Singapour & 6,9 & 17,040 \\
26 & Oakland / USA & 6,8 & 1,777 \\
27 & Manille / Philippines & 6,6 & 2,289 \\
28 & Yokohama / Japon & 6,6 & 2,317 \\
29 & Kaohsiung / Taïwan & 6,3 & 7,426 \\
30 & New-York / USA & 6,3 & 3,006 \\
\hline & & &
\end{tabular}

Source : Container Management, mai 2001, n 192, p. 35

24 L'arrivée de méga porte-conteneurs pousse les armements impliqués à s'emparer du marché des produits en vrac et des néo-vracs. Les habitudes des courtiers spécialistes des vracs vont s'en trouver modifiées si la migration vers les lignes conteneurisées s'affirme. Un courtier londonien, dans un entretien paru dans la livraison de Fairplay Weekly du 10 juin 1999 estimait alors que le pourcentage des échanges mondiaux de marchandises sèches transportées en conteneurs était passé de 5,9\% en 1980 à plus de $25 \%$. Du fait de l'augmentation importante de la capacité de la flotte de porte-conteneurs, les échanges conteneurisés prennent annuellement entre 14 à $21 \mathrm{M} \mathrm{T}$ de marchandises par an au secteur conventionnel. Le niveau d'intrusion des conteneurs dans le marché des vracs peut atteindre jusqu'à $3 \%$ de la demande de vraquiers handymax (10 000 à 45000 tpl), ce qui, en conséquence peut porter sur un pourcentage non négligeable du marché de l'affrètement.

La croissance des flux conteneurisés se marque par un doublement des tonnages de 249 MT en 1990 à 509 MT en 1999. Durant cette période la flotte de porte-conteneurs a augmenté à hauteur de $10 \%$ par an, et en 1999, sa capacité représente, selon les estimations, l'équivalent du quart de la flotte de vraquiers secs. Il existe une corrélation entre l'évolution des deux flottes. Des porte-conteneurs ont été construits pour remplacer des vraquiers âgés à mesure que se développait le mouvement de conteneurisation des vracs mineurs.

Les vracs mineurs que la conteneurisation vise ne sont pas le charbon ou les minerais. Dans la dernière décennie du $\mathrm{XX}^{\mathrm{e}}$ siècle, les échanges maritimes de vracs mineurs ont augmenté de $11 \%$ environ. En dépit de la baisse cyclique des taux de fret concernant le 
transport à bord des handymax, la concurrence des porte-conteneurs vient du fait que les taux du transport conteneurisé ont baissé encore plus que dans le secteur du vrac avec la surcapacité liée à l'arrivée de navires de plus en plus grands. Le phénomène a été accéléré par la crise des économies asiatiques à la fin de la décennie 1990: les pays d'Asie exportent bien plus qu'ils n'importent d'où un déséquilibre pour remplir les boîtes et la tendance à baisser le prix du transport, ce qui devient attractif pour les petits vracs. Selon Fairplay Weekly du 10 juin 1999, le coût unitaire moyen journalier d'un 20 pieds est passé de 13,30 \$ en 1992 à 8,20 \$ en 1998. Le coût du transport d'une marchandise conteneurisée est déterminé par le coût marginal du système maritime intermodal, et non par le coût entier de la manutention du vrac.

Certains vracs tels que les céréales peuvent être conteneurisés. Celles-ci ne sont pas comme le charbon et les minerais une marchandise qui ne peut convenir qu'au transport par vraquiers. Elles voyagent de milliers de fermes vers une centaine de pays, alors que charbons et minerais vont d'une douzaine de mines vers deux douzaines de ports. Là où charbons et minerais remplissent les cales d'un vraquier et sont transportés à un taux similaire au coût d'extraction, les céréales doivent être stockées lors des pics saisonniers à un coût élevé. Charbons et minerais sont de façon générale des produits assez homogènes par nature, on ne rencontre pas trop de problème de mélanges. Les céréales, en revanche présentent des variétés de qualités différentes. Ainsi il existe quatre grandes variétés de blé états-uniens qui ne peuvent pas être mélangées. Si le transport de vracs doit prendre en compte trop de variétés, il devient moins efficace et moins compétitif. Ainsi il n'est pas rare que l'Australie exporte des cargaisons conteneurisées jusqu'à 50000 $\mathrm{T}$ de céréales. De son côté l'office canadien des blés loue chaque année des milliers de conteneurs. Au printemps 1999, AP Moller a traité un transport de 40000 T de céréales dans le cadre du programme mondial contre la faim, sur la base de $2000 \mathrm{~T}$ hebdomadaires (soit 100 conteneurs de 20 pieds). Ce sont là quelques exemples d'une tendance lourde.

Le transport des vracs mineurs, c'est-à-dire le sucre, les engrais, l'acier (par exemple sous forme de coils), les ferrailles, le cacao, les produits forestiers, etc. pose un problème aux armateurs de vraquiers du fait de l'irrégularité avec laquelle ils sont transportés sous une forme brute. Or aujourd'hui les pays en développement exportateurs cherchent à valoriser leurs productions. Les produits forestiers de la COA sont de plus en plus conteneurisés. Pour des produits comme le cacao, le conteneur assure une protection au chargement, au déchargement et l'on peut introduire des agents hydroscopiques dans la boîte aérée durant le transport.

L'idée que la conteneurisation des vracs est marginale, que la conteneurisation ne convient qu'aux marchandises chères est assez répandue. Or cette idée méconnaît l'impact de la conteneurisation: déjà dans les années 1980 les transports réfrigérés ont cédé des parts de marché aux lignes conteneurisées (selon le consultant Drewry, en 2000, la moitié des échanges sous froid s'effectue par les porte-conteneurs). Les modernes porte-conteneurs ont à bord des conteneurs frigorifiques. Ceux-ci coûtent chers et les armements ont attiré la marchandise en offrant des taux attractifs au détriment des navires frigorifiques traditionnels.

30 C'est maintenant au tour des transports de vracs. Le grand problème des filières conteneurisées est d'avoir à gérer le déséquilibre des flux et le repositionnement des conteneurs vides. Si les vracs peuvent contribuer à remplir les boîtes, les lignes régulières vont se battre pour ce marché, et d'autant plus vigoureusement si les TGPC entrent en flotte. 
31 Les conséquences commerciales de l'arrivée de ces méga porte-conteneurs reposent notamment sur le fait que le phénomène de la conteneurisation englobe des types de marchandises de plus en plus larges. Ainsi à la mise en place de tels navires se manifestera vraisemblablement un effet " aspirateur ", ce que l'on a déjà pu observer lors de l'entrée en flotte de générations successives de porte-conteneurs. Cet effet se traduit par l'aspiration des frets dans la région avec une conséquence négative sur le marché. Ceci est partiellement compensé par la réduction du coût des cellules. Savoir si cette réduction est suffisante dépend de l'état du marché.

L'effet des taux de fret très faibles que les opérateurs de TGPC pourront proposer conduit à la conteneurisation des vracs et néo-vracs. Une fois l'habitude prise par les chargeurs, ceux-ci vont continuer à utiliser la conteneurisation surtout si les taux de fret restent attractifs, c'est-à-dire bas.

$\mathrm{Au}$ total, en 2000, la pénétration de la conteneurisation représente $30 \%$ du marché des marchandises sèches, soit $600 \mathrm{Mt}$. Les prévisions, selon Drewry Consultant (Fairplay Weekly du 18 avril 2002), annoncent un taux de pénétration de $40 \%$ en 2005 (800 Mt).

L'arrivée des TGPC aura ainsi des impacts sur le marché du transport maritime dans son ensemble, y compris sur l'affrétement des navires vraquiers perturbé par les conséquences de la conteneurisation des vracs.

Une autre conséquence, mais propre au marché de la flotte de porte-conteneurs, est celle de la gestion du nombre nécessairement plus important de boîtes en circulation.

Des plans existent pour construire des navires de 15000 à 18000 boîtes. S'ils sont effectivement construits, les problèmes commerciaux liés au remplissage des boîtes ne seront pas les seuls à se poser, les ports seront confrontés au problème de l'adaptation de leurs équipements aptes à servir de tels monstres au bénéfice des transporteurs maritimes et alors que ces équipements, en général, sont financés par le contribuable.

\section{Les méga porte-conteneurs et les infrastructures portuaires}

37 A partir du moment où des porte-conteneurs de 15000 EVP sont techniquement plausibles il s'agit de réfléchir à leur utilisation en matière commerciale. Il est possible de construire un porte-conteneurs suezmax de $400 \mathrm{~m}$ de long, mais il est plus difficile d'organiser une rotation concernant par exemple 4 ports d'escale entre les Amériques, l'Europe et l'Asie. La question des accès nautiques, des limites des portiques se pose. De nouveaux méga-ports desservant un continent, par exemple un terminal géant situé dans les Caraïbes ou en Amérique centrale, pourront-ils offrir un service plus compétitif que celui des services directs? Peut-on imaginer un tel méga-hub en Asie desservant les territoires du Japon aux Indes? Singapour peut-il avoir une telle ambition ? Ou en Europe Algésiras? Le passage portuaire doit être à la hauteur des gains obtenus en mer avec les TGPC.

\section{Le concept de coopération entre ports voisins}

Beaucoup de pays ont préparé leurs ports à l'essor de la conteneurisation en privatisant tout ou partie des quais (Baird, 1999, 2001). Ainsi se sont développés les grands opérateurs de terminaux de la 4è génération (Lacoste, Terrassier, 2001) parallèlement à la mise en 
place des Alliances (Cariou, 2000) dont les grands armements opèrent des terminaux dédiés. Ils constituent des réseaux de ports implantés dans des zones à fort potentiel.

Mais ces très grands ports à conteneurs ne sont pas toujours les plus attractifs en matière de taxes portuaires et l'accueil des TGPC suppose de nouvelles installations adaptées, or, en général, la place manque. De là peut-être, la naissance d'un nouveau concept.

Un porte-conteneurs de $12000 \mathrm{EVP}$ ne peut avoir accès actuellement qu'à une quinzaine de ports dans le monde offrant une profondeur d'au moins $15 \mathrm{~m}$. Le dragage à des profondeurs de ce type va devenir inévitable ce qui pose un problème environnemental, d'autant que l'extension spatiale des terminaux, pour répondre aux besoins des TGPC, n'est pas possible partout. On peut alors imaginer des accords de coopération (Heaver, 2001) remplaçant, à terme, la situation de compétition féroce qui sévit aujourd'hui, une association entre d'une part un grand port performant, mais bloqué dans son développement, efficace et cher, et, d'autre part, un port voisin au coût attractif et offrant l'espace nécessaire à l'accueil d'un volume accru de boîtes.

Il existe des possibilités de coopérer plus que d'entrer en compétition pour des ports dans différentes régions du monde notamment en Europe et en Asie (Dong-Wook Song, 2003). Hong Kong, après trente années de forte croissance, connaît une certaine stagnation alors que les ports chinois voisins de Yantian, Shekou et Chiwan connaissent de fortes progressions : le trafic de Hong Kong recule de 18,1 à 17,8 M EVP entre 2000 et 2001 alors que celui de Shenzen augmente de $27 \%$ atteignant 5,05 M EVP en 2001. Ceci s'explique pour des raisons de coûts portuaires plus élevés à Hong Kong et des difficultés de desserte de l'hinterland avec ces longues queues de véhicules, de camions à destination de Guangzhou bloqués dans les embouteillages de l'agglomération. Une coopération qui ne sera évidemment pas simple à concevoir pourrait s'organiser afin de répondre aux besoins des futurs TGPC.

Différents auteurs ont montré ce phénomène des complémentarités portuaires (Hyzy, 1999, Juhel, 2000). Pour rationaliser l'espace et les investissements l'idée de coopération portuaire fait son chemin, mais ce processus reste un fait isolé (cf. la fusion entre les ports de Malmö et de Copenhague).

\section{Les TGPC, les contraintes nautiques pour les ports, le nouveau concept de terminal à manutention babord-tribord}

L'arrivée des TGPC de 15000 EVP va remettre en cause l'organisation de la filière de transport. Ces nouveaux over-panamax voire ces Malacca-Max de 18000 EVP n'escaleront que dans un nombre très réduit de ports aptes à les recevoir sur le plan nautique et à traiter la marchandise. Les contraintes sont fortes pour les ports.

Tous les grands ports sont conduits à revoir sans cesse les accès nautiques pour suivre la course au gigantisme naval.

Le port de New-York/New-Jersey a engagé de grands travaux de dragages du Kill van Kull et des accès aux grands terminaux afin de recevoir des 7000 boîtes à l'horizon 2005 . Rotterdam avec ses 16,65 m de la Maasvlakte accueille déjà cette catégorie de navires et pourra recevoir les très grands porte-conteneurs du futur. Mais Anvers n'offre que $13 \mathrm{~m}$ de tirant d'eau et Hambourg $12 \mathrm{~m}$. Les longs et coûteux travaux de dragage et d'amélioration des conditions techniques, à terre, sans même évoquer les contraintes environnementales, pourront-ils être rentabilisés aisément? Plutôt que d'escaler dans les 
ports traditionnels, les armements conteneurisés choisissent des plateformes de transbordement, des hubs, offrant de bonnes facilités d'accès.

Le succès des hubs méditerranéens bien placés sur la route Est-Ouest comme Marsaxlokk ouvert en 1990 et Gioia Tauro ouvert en 1995 montre une tendance lourde (Elliot, 2001; Juhel, 1999) confirmée par la création récente des hubs de Tarente et de Cagliari. D'autres exemples pourraient être cités ailleurs comme Freeport aux Bahamas qui traite $1 \mathrm{M}$ EVP par an depuis son extension de 1999. Mais l'arrivée des TGPC requiert des installations d'une autre ampleur.

Peu de ports actuellement sont en mesure d'alimenter ces leviathans des mers avec leurs 22 à 24 rangées de boîtes en largeur. Le déploiement de porte-conteneurs de 15000 EVP sur les grandes routes Est-Ouest avec les ramifications Nord-Sud assurées par des feeders de 250 à 6000 EVP suppose de nouveaux ports. Ceux-ci devraient se situer en Asie du SudEst, à la sortie (entrée) occidentale de la Méditerranée (actuellement, Algésiras est un des rares hubs existants a pouvoir assurer la fonction de méga-hub), dans les Caraïbes et sur la côte ouest de l'Amérique du Nord. Un tel méga-hub devrait pouvoir offrir des postes à quai pour des $15000 \mathrm{EVP}$, pour de grands feeders et pour de petits feeders.

Il est peu probable qu'un service de TGPC puisse se mettre en place sans une escale étatsunienne à chaque extrémité du fait de la puissance commerciale et économique du continent nord-américain. Et pour faire face à la compétition des services directs, il sera très difficile de desservir le range Tokyo - Mumbai avec une escale seulement, ce qui implique une escale orientale au Japon ou à Taïwan, et une escale occidentale à Singapour, Port Klang ou Colombo.

La congestion des terminaux dans beaucoup de ports notamment en Europe, les difficultés d'extension du fait des contraintes environnementales des législations, poussent à la création de nouveaux hubs illustrant le concept de terminaux flottants, le navire étant opéré par ses deux flancs.

50 L'idée n'est pas totalement nouvelle. Le port de Hong Kong utilise déjà la zone de mouillage pour manutentionner, entreposer des conteneurs sur des plateformes flottantes. Cela pose évidemment le problème de la sécurité sur les plans d'eau qui sont parfois congestionnés.

51 Le concept de terminal flottant découle de la difficulté d'opérer rapidement les TGPC d'un seul côté.

52 Avec 24 boîtes dans la largeur du navire, il sera nécessaire de prévoir l'utilisation de portiques de chaque côté du navire. Mais aucun port ne voudra se lancer dans l'aménagement de doubles quais qui seront obsolètes lorsque les navires seront encore plus grands. La solution, mais à quel coût, pourrait être des quais flottants, et même un terminal entier flottant. On imagine aisément le coût élevé des protections à prendre pour de telles installations si elles sont situées par exemple dans un secteur traversé par les cyclones comme dans les Caraïbes ou le Pacifique occidental.

53 Ceci n'est qu'un aspect du problème, celui du chargement et du déchargement du navire. L'autre aspect est la mise en place d'une logistique capable de traiter la masse de marchandises en entrée et en sortie des terminaux. Ceux-ci, d'après certains experts, devraient être capables de traiter 330 boîtes à l'heure. De là l'idée de créer des méga-hubs off-shore alimentés par des feeders.

54 L'arrivée des TGPC, si elle risque de renforcer les grands hubs existants capables de s'adapter en conséquence, va vraisemblablement provoquer la construction de nouvelles 
plates-formes de transbordement notamment offshore, le concept de l'instrument nautique nouveau requérant un nouveau concept de ports.

\section{Le concept de nouveaux hubs en eau profonde}

a défini avec l'opérateur ECT, le projet FAMAS (First All Modes All Sizes). Le but de ce projet est de développer, dans un partenariat public - privé, une nouvelle génération de terminaux à conteneurs et de centres de services logistiques dans le cadre de la Maasvlakte 2. En 2002, le gouvernement néerlandais a donné l'autorisation nécessaire à la construction de cette extension de la Maasvlakte. Le projet prévoit que 3000 ha soient aménagés sur la mer. En compensation une réserve de 750 ha sera créée dans la zone du Rijnmond Rotterdam. Ainsi en 2020, le volume de conteneurs transitant par Rotterdam serait de 8,7 M EVP sur la Maasvlakte 1 et autant sur la Maasvlakte 2, celle-ci étant constituée de 3 môles (et non un quai unique allongé).

61 La tendance, avec l'arrivée de navires de plus en plus grands est aussi à l'utilisation de hubs offshore.

Le concept hub and spokes est le plus performant face au concept de port classique pour traiter les grands navires. Les coûts d'amortissement d'un géant de 7000 à 10000 boîtes coûtant de $70 \mathrm{M}$ à $100 \mathrm{M} \$$ ou environ 10000 \$ par EVP diminuent naturellement avec la rapidité des rotations et l'étalement des frais fixes par les gains des voyages successifs. Il peut être jugé plus économique de ne pas conduire les navires jusqu'aux hubs continentaux, et d'imaginer une plate-forme offshore à partir de laquelle se ferait la redistribution des boîtes. Les Amériques avec les Caraïbes, l'Asie, la Méditerranée ont des sites favorables ; en Europe du Nord-Ouest, rares sont les sites propices. 

Flow permet d'accomoder des navires de $20 \mathrm{~m}$ de tirant d'eau. Depuis 1977 le terminal Flotta exporte un flux régulier de pétrole dans des aframax; il est approvisionné par pipe-line des champs pétroliers offshore de la mer du Nord. La production a atteint jusqu'à 300000 barils par jour, mais elle n'est plus que la moitié de cela, et il s'agit pour les autorités de penser à l'après-pétrole.

Plusieurs structures se sont associées, Scotland's Highlands and Islands enterprise (HIE), Orkney Islands Council (OIC)... pour proposer d'établir un hub à conteneurs sur l'île de Flotta à Scapa Flow, pour répondre aux trafics transatlantiques et en association avec Halifax en Nouvelle-Ecosse, qui a l'avantage d'être également un port en eau profonde. Le projet porte sur la construction de $3 \mathrm{~km}$ de quais aptes à manutentionner $4 \mathrm{M} \mathrm{EVP} \mathrm{par} \mathrm{an}$ pour un coût de $686 \mathrm{M} \$$. Le transbordement représentera environ $95 \%$ des trafics, des feeders de 1000 à 2500 EVP feront les trajets terminaux ou initiaux de ou vers les grands ports continentaux du Northern Range, des feeders plus petits desservant les pays de la Baltique et les Iles Britanniques.

Plusieurs compagnies sont intéressées comme Forth Ports, Clydeport, et une compagnie locale, Scapa Terminal. La meilleure structure pour réussir serait d'associer un opérateur portuaire, un armement maritime et une société de développement local. Des grands armements européens américains ou asiatiques pourraient s'intéresser au projet par le biais d'un terminal dédié.

Un centre de transbordement à Scapa Flow offrira une économie d'échelle aux chargeurs et présente des avantages sur le plan environnemental par rapport aux autres grands ports congestionnés qui doivent engager des travaux de dragage. Une étude menée par le Maritime Research Unit de l'université Napier d'Edimbourg sur le projet montre que le coût du dragage du chenal de l'Escaut ou de l'Elbe revient à $12 \$$ par EVP, ce qui est absurde s'agissant des très grands porte-conteneurs.

La situation nordique du terminal et les conditions climatiques difficiles peuvent être un handicap au projet, mais Scapa Flow bénéficie de l'influence du Gulf Stream et est placé sur la route la plus courte entre l'Amérique et l'Europe. Les études faites par le Maritime Research Unit d'Edimbourg montre, pour l'armateur, le gain de temps (donc d'argent) induit par l'escale dans le hub de Scapa Flow. On arrive à un total de 11 à 12 jours quelle que soit la combinaison des ports touchés sur le continent alors que le transit actuel est de l'ordre de 14 jours.

\section{Conclusion}

L'arrivée des TGPC va avoir des conséquences dans de nombreux domaines, celui du transport maritime en général et celui de la filière conteneurisée en particulier. Alors que jusque là, depuis la spécialisation des navires à partir du milieu du $\mathrm{XX}^{\mathrm{e}}$ siècle, l'organisation et la gestion de la navigation dans le domaine des vracs et dans celui des divers était distincte, désormais, les vracs entreront de plus en plus dans les filières conteneurisées avec l'arrivée des TGPC et toutes les conséquences que cela peut avoir sur le marché global. A l'échelle du marché du transport conteneurisé, c'est l'ensemble de la filière qui devra s'adapter; la hiérarchisation portuaire va se trouver modifiée, elle dépendra de la coopération entre les grands armements lancés dans la course au gigantisme et les opérateurs portuaires obligés de se lancer aussi dans des 
investissements de plus en plus lourds. Le système de grands hubs déconnectés de la terre va se trouver stimulé, le transport devient de plus en plus océanique plus que maritime, mais on assiste conjointement à la territorialisation des espaces marins ; avec des platesformes portuaires situés dans la ZEE, la déconnexion de la ville portuaire et des terminaux est totale.

\section{BIBLIOGRAPHIE}

ALIX Y., SLACK B., COMTOIS C. (1999), « Alliance or acquisitions? Strategies for growth in the container shipping industry, the case of C.P. Ships ", Journal of transport geography, 7, pp. 203-208.

BAIRD A.J. (2001), « Trends in port privatisation », Ports \& Harbors, 46, 1, pp. 16-21.

BAIRD A. J. (1999), «Container vessels of the next generation: are seaport ready to face the challenge », Ports \& Harbors, 44, 7, pp. 15-23.

BODY H. (2000), « Battling for box growth », Container Management, 181, pp. 39-44.

CAI C. (2002), « Port development in China at present and in the future », Ports \& Harbors, 47, 5, pp. 12-17.

CARIOU P. (2000), Les alliances stratégiques dans le transport maritime de lignes régulières : efficacité ou pouvoir de marché ?, Thèse de Doctorat en économie, Université de Nantes, 325 p.

DARCHE M. (2002), « Trends in container transport: the modal split », Ports \& Harbors, 47, 8, pp. 14-16.

DONG-WOOK SONG (2003), « Port co-operation in concept and practice », Maritime Policy \& Management, 30, 1, pp. 29-44.

ELLIOT N. (2001), « Hub port Italia », Port Development International, 17, 4, pp. 26-27.

GENET B. (1999), «Conteneurs et nouvelle géographie portuaire », Hérodote, 93, pp. 134-143.

HEAVER T. (2001), « Co-operation and competition in international container transport: strategies for ports », Maritime Policy \& Management, 28, 3, pp. 293-305.

HYZY V. (1999), « De la Baltique à la Méditerranée : le développement des complémentarités portuaires », Acta Geographica, 119, pp. 43-57.

JUHEL M.H. (2000), « Globalisation and partnerships in ports trends », Ports \& Harbors, 45, 5, pp. 9-14.

JUHEL M.H. (1999), « Global challenges for ports and terminals in the new area », Ports \& Harbors, 44, 2, pp. 17-27.

LACOSTE R., TERRASSIER N. (2001), « La manutention portuaire conteneurs : les opérateurs internationaux - perspectives européennes ", Synthèse ISEMAR, 39, 4 p.

LOPEZ PONTON E. (2003), « Les porte-conteneurs géants : mythe ou réalité », Synthèse ISEMAR, 52, $4 \mathrm{p}$. 
MAS S. (2001), « Les ports conteneurisés de nouvelle génération : critères d'attractivité, enjeux et difficultés à surmonter », Transports, 410, pp. 426-435.

MUSSO E., FERRARI C, BENACCHIO M. (1999), « On the global optimum size of ports terminals », International Journal of Transport Economics, 26, 3, pp. 415-437.

NOTTEBOOM T. (2002), "Current issues in port logistic and intermodality », Institut of Transport and Maritime Management, Anvers, Garant, $158 \mathrm{p}$.

NUMAN J. (2001), « The growth group », Container Management, 192, pp. 34-38.

NEUMEISTER M. (2001), « Transport conteneurisé : les ports asiatiques aux quatre premières places ", Journal de la Marine Marchande, 4274, pp. 20-41.

SLACK B., COMTOIS C.,SLETMO G. (1996), « Shipping lines as agents of change in the port industry ", Maritime Policy \& Management, 3, pp. 289-300.

VAN DEN BOSSCHE B. (2000), «Quels sont les avantages et inconvénients des terminaux dédiés? » Le Lloyd, 42382, pp. 1-3.

\section{RÉSUMÉS}

La tendance à l'utilisation de porte-conteneurs de plus en plus grands est liée à la croissance des échanges maritimes internationaux. Si les limites techniques en matière de construction navale ne sont pas un obstacle, les conséquences sur l'organisation même du transport maritime sont réelles : la surcapacité induite par le volume accru de cale va inciter les armements à capter de nouveaux types de marchandises dans le domaine des vracs et néo-vracs, ainsi qu'à gérer de façon plus efficace le repositionnement des conteneurs. Un nouveau concept de terminal devra être défini, placé en eau profonde sur le littoral d'un continent ou en situation insulaire. De nouveaux types de relations interportuaires sont à envisager avec l'introduction, dans un contexte très concurrentiel, de politiques de coopération entre ports.

The trend of the use of always bigger containerships is linked with the growth of the maritime exchanges. If the technological limits in matter of shipbuilding are not an impediment, the consequences upon the management of maritime transport, most particularly the Alliances, are substantial. The overcapacity will instigate the shipping companies to attract new categories of goods within the sector of the bulks and neo-bulks, and also to manage the positioning of the boxes better. A new concept of terminal is to be defined situated in deep water on the coast of a continent or offshore on an island. New types of port relationships must be set up with the introduction of politics of port cooperation within the usual very competitive context.

\section{INDEX}

Mots-clés : méga porte-conteneurs, surcapacité, vrac, coopération portuaire, terminaux du futur, nouveaux hubs

Keywords : mega containerships, overcapacity, bulk, port cooperation, terminal of the future, new hubs 


\section{AUTEUR}

\section{JACQUES MARCADON}

Université de Nantes - UMR 6554 LETG du CNRS, jacques.marcadon@humana.univ-nantes.fr 